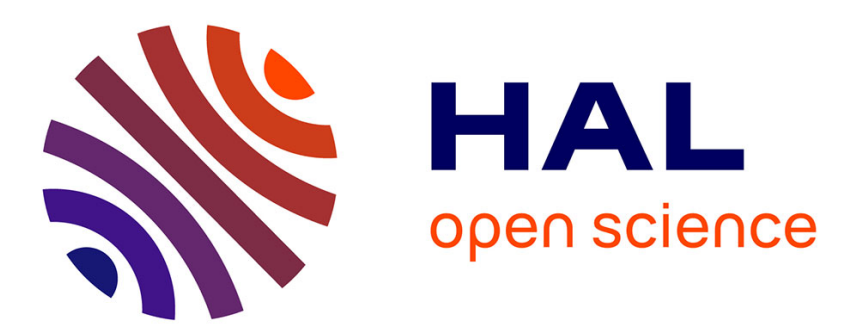

\title{
Canonical decomposition of dichotomous basic belief assignment
}

\author{
Jean Dezert, Florentin Smarandache
}

\section{To cite this version:}

Jean Dezert, Florentin Smarandache. Canonical decomposition of dichotomous basic belief assignment. International Journal of Intelligent Systems, 2020, 35 (7), pp.1105-1125. 10.1002/int.22236 . hal-02873613

\section{HAL Id: hal-02873613 https://hal.science/hal-02873613}

Submitted on 18 Jun 2020

HAL is a multi-disciplinary open access archive for the deposit and dissemination of scientific research documents, whether they are published or not. The documents may come from teaching and research institutions in France or abroad, or from public or private research centers.
L'archive ouverte pluridisciplinaire HAL, est destinée au dépôt et à la diffusion de documents scientifiques de niveau recherche, publiés ou non, émanant des établissements d'enseignement et de recherche français ou étrangers, des laboratoires publics ou privés. 


\title{
Canonical Decomposition of Dichotomous Basic Belief Assignment
}

\author{
Jean Dezert, ${ }^{1, *}$ Florentin Smarandache ${ }^{2, \dagger}$ \\ 1 ONERA - The French Aerospace Lab, \\ Chemin de la Hunière, F-91761 Palaiseau, France. \\ 2 Department of Mathematics, \\ Univ. of New Mexico, Gallup, NM, USA.
}

\begin{abstract}
In this paper, we prove that any dichotomous basic belief assignment (BBA) $m$ can be expressed as the combination of two simple belief assignments $m_{p}$ and $m_{c}$ called respectively the pros and cons BBAs thanks to the proportional conflict redistribution rule no 5 (PCR5). This decomposition always exists and is unique and we call it the canonical decomposition of the BBA $m$. We also show that canonical decompositions do not exist in general if we use the conjunctive rule, the disjunctive rule, Dempster's rule, Dubois \& Prade's or Yager's rules, or even the averaging rule of combination. We give some numerical examples of canonical decompositions and discuss of the potential interest of this canonical decomposition for applications in information fusion.
\end{abstract}

\section{INTRODUCTION}

The belief functions (BF) introduced by Shafer in the mid of 1970's ${ }^{1}$ from Dempster's works are well known and used in the artificial intelligence community to model epistemic uncertainty and to reason with it for information fusion. In Dempster-Shafer theory, the combination of basic belief assignments (BBAs) provided by distinct sources of evidence is done with Dempster's rule of combination which suffers of serious drawbacks in high conflict situation as discussed by Zadeh ${ }^{16,17}$, but also in very low conflict situations ${ }^{4}$. As a matter of fact many rules of combination have been proposed in the literature $^{2}$ (Vol. 2), among them the combination of two sources of evidence based on the proportional conflict redistribution principle no5 (PCR5 rule) ${ }^{8}$ has been shown successful in applications, and well justified theoretically. However its complexity remains one of its limitations to prevent its use in large fusion problems.

\footnotetext{
*email : jean.dezert@onera.fr

†email : smarand@unm.edu
} 
In this work we show how the fusion of dichotomous BBAs could be done thanks to their PCR5-based canonical decomposition which is always possible. Such decomposition of dogmatic or nondogmatic BBA has never been presented in the literature so far. Only a canonical decomposition based on conjunctive rule involving improper BBA has been proposed by Smets in $1995^{3}$ and extended later by Denœux ${ }^{12}$ to develop the cautious rule of combination. Here the canonical decomposition we present is done differently, and we show that any dichotomous BBA is always the result of the PCR5 fusion of a simple proper pro-evidence BBA $m_{p}$ with a simple proper contra-evidence BBA $m_{c}$, and we show that this decomposition is unique.

This paper is organized as follows. After a brief recall of basics of belief functions in section 2, we present the canonical decomposition problem (CDP) in section 3 and we show the impossibility to realize the CDP of a non dogmatic BBA with conjunctive rule, disjunctive rule, Yager's and Dubois \& Prade rules, and even with the averaging rule of combination. In section 4, we analyze the CDP based on Dempster's rule of combination and we show that it cannot be done for a dogmatic BBA. In section 5, we prove that the canonical decomposition based on PCR5 rule always exist for all the cases. In section 6, we present some particular decompositions of a dichotomous BBA (including dogmatic BBA). Some numerical examples are presented in section 7, and potential interests of this PCR5-based canonical decomposition are discussed in section 8 . The last section concludes this paper and opens a challenging question for application of this new approach.

\section{BASICS OF BELIEF FUNCTIONS}

Belief functions (BF) have been introduced by Shafer in ${ }^{1}$ to model epistemic uncertainty. We assume that the answer ${ }^{1}$ of the problem under concern belongs to a known (or given) finite discrete frame of discernment (FoD) $\Theta=\left\{\theta_{1}, \theta_{2}, \ldots, \theta_{n}\right\}$, with $n>1$, and where all elements of $\Theta$ are mutually exclusive ${ }^{2}$. The set of all subsets of $\Theta$ (including empty set $\emptyset$ and $\Theta$ ) is the power-set of $\Theta$ denoted by $2^{\Theta}$. A proper Basic Belief Assignment (BBA) associated with a given source of evidence is defined ${ }^{1}$ as a mapping $m(\cdot): 2^{\Theta} \rightarrow[0,1]$ satisfying $m(\emptyset)=0$ and $\sum_{A \in 2^{\Theta}} m(A)=1$. In some BF related frameworks, like in Smets Transferable Belief Model (TBM) ${ }^{3}, m(\emptyset)$ is allowed to take a positive value. In this case, $m(\cdot)$ is said improper because it doesn't satisfy Shafer's definition ${ }^{1}$. The quantity $m(A)$ is called the mass of $A$ committed by the source of evidence. Belief and plausibility functions are respectively defined from a proper BBA $m(\cdot)$ by

$$
\operatorname{Bel}(A)=\sum_{B \in 2^{\Theta} \mid B \subseteq A} m(B)
$$

and

$$
P l(A)=\sum_{B \in 2^{\Theta} \mid A \cap B \neq \emptyset} m(B)=1-\operatorname{Bel}(\bar{A}) .
$$

where $\bar{A}$ is the complement of $A$ in $\Theta$.

\footnotetext{
${ }^{1}$ i.e. the solution, or the decision to take.

${ }^{2}$ This is so-called Shafer's model of FoD ${ }^{2}$.
} 
$\operatorname{Bel}(A)$ and $\operatorname{Pl}(A)$ are usually interpreted respectively as lower and upper bounds of an unknown (subjective) probability measure $P(A) . A$ is called a Focal Element (FE) of $m(\cdot)$ if $m(A)>0$. When all focal elements are singletons then $m(\cdot)$ is called a Bayesian $B B A^{1}$ and its corresponding $\operatorname{Bel}(\cdot)$ function is equal to $P l(\cdot)$ and they are homogeneous to a (subjective) probability measure $P(\cdot)$. The vacuous BBA, or VBBA for short, representing a totally ignorant source is defined $\operatorname{as}^{3} m_{v}(\Theta)=1$. A dogmatic BBA is a BBA such that $m(\Theta)=0$. If $m(\Theta)>0$ the BBA $m(\cdot)$ is nondogmatic. A simple $\mathrm{BBA}$ is a BBA that has at most two focal sets and one of them is $\Theta$. A dichotomous non dogmatic mass of belief is a BBA having three focal elements $A, \bar{A}$ and $A \cup \bar{A}$ with $A$ and $\bar{A}$ subsets of $\Theta$.

In his Mathematical Theory of Evidence ${ }^{1}$, Shafer proposed to combine $s \geq 2$ distinct sources of evidence represented by BBAs $m_{1}(),. \ldots, m_{s}($.$) over the same FoD$ $\Theta$ with Dempster's rule (i.e. the normalized conjunctive rule). The justification and behavior of Dempster's rule have been disputed over the years from many counterexamples involving high and low conflicting sources (from both theoretical and practical standpoints) as reported in ${ }^{4-7}$.

Many rules of combination exist in the literature ${ }^{4}$, among them we recommend the rule based on the proportional conflict redistribution principle no5 (PCR5 rule) ${ }^{8}$ which has been shown successful in applications and well justified theoretically. That is why we analyze it in details for solving the BF canonical decomposition problem (BF-CDP). PCR5 transfers the conflicting mass only to the elements involved in the conflict and proportionally to their individual masses, so that the specificity of the information is entirely preserved in this fusion process. (see ${ }^{2}$, Vol. 2 and Vol. 3 for full justification and examples). The PCR5 combination of two BBAs $m_{1}$ and $m_{2}$ defined on the same FoD $\Theta$, denoted by $m_{P C R 5}=\operatorname{PCR} 5\left(m_{1}, m_{2}\right)$, is mathematically defined as $m_{P C R 5}(\emptyset)=0$ and $\forall X \in 2^{\Theta} \backslash\{\emptyset\}$

$$
\begin{aligned}
m_{P C R 5}(X)=\sum_{\substack{X_{1}, X_{2} \in 2^{\Theta} \\
X_{1} \cap X_{2}=X}} m_{1}\left(X_{1}\right) m_{2}\left(X_{2}\right)+ \\
\qquad \sum_{\substack{X_{2} \in 2^{\ominus} \\
X_{2} \cap X=\emptyset}}\left[\frac{m_{1}(X)^{2} m_{2}\left(X_{2}\right)}{m_{1}(X)+m_{2}\left(X_{2}\right)}+\frac{m_{2}(X)^{2} m_{1}\left(X_{2}\right)}{m_{2}(X)+m_{1}\left(X_{2}\right)}\right]
\end{aligned}
$$

where all denominators in (3) are different from zero. If a denominator is zero, that fraction is discarded. The properties of PCR5 can be found in ${ }^{9}$. Extension of PCR5 for combining qualitative BBA's can be found in ${ }^{2}$, Vol. $2 \& 3$. All propositions/sets are in a canonical form. A variant of PCR5, called PCR6 has been proposed by Martin and Osswald in ${ }^{2}$, Vol. 2, for combining $s>2$ sources. The general formulas for PCR5 and PCR6 rules are also given in ${ }^{2}$, Vol. 2. PCR6 coincides with PCR5 when one combines two sources. The difference between PCR5 and PCR6 lies in the way the proportional conflict redistribution is done as soon as three (or more) sources are involved in the fusion. From the implementation point of view, PCR6 is simpler to implement than PCR5. For convenience, very basic (not optimized) Matlab codes of PCR5 and PCR6

\footnotetext{
${ }^{3}$ The complete ignorance is denoted $\Theta$ in Shafer's book ${ }^{1}$.

${ }^{4} \mathrm{see}^{2}$, Vol. 2 for a detailed list of fusion rules.
} 
fusion rules can be found in ${ }^{2,10}$ and from the toolboxes repository on the web ${ }^{11}$. In the sequel we work with PCR5 rule because only two BBAs are involved in the canonical decomposition process we present.

\section{THE CANONICAL DECOMPOSITION PROBLEM}

We consider a dichotomous (simplest) FoD $\Theta$ made of only two exclusive elements $A$ and $\bar{A}$, that is $\Theta=\{A, \bar{A}\}$ and we consider a given $\operatorname{proper}^{5} \mathrm{BBA} m(\cdot): 2^{\Theta} \rightarrow[0,1]$ of the form

$$
m(A)=a, \quad m(\bar{A})=b, \quad m(A \cup \bar{A})=1-a-b
$$

with $0<a<1,0<b<1$ and $a+b<1$.

The conditions $0<a<1$ and $0<b<1$ mean that $A$ and $\bar{A}$ are focal elements of the BBA. The restriction $a+b<1$ means that the BBA is nondogmatic. This assumption of nondogmaticity of the BBA $m(\cdot)$ can be justified because most (if not all) states of belief, being based on imperfect and not entirely conclusive evidence, should be represented by nondogmatic belief functions, even if the mass $m(\Theta)$ is very small as argued by Denœux in ${ }^{12}$ (p. 240). In fact, we can always slightly modify a dogmatic BBA $m(\cdot)$ in a nondogmatic BBA by discounting it with some small discount rate $\epsilon>0$ and letting $\epsilon$ tend towards $0^{3}$. The case of dogmatic belief, as well as degenerate cases with $a=0$ and $b=0$ will be discussed in Section 6. Note that his assumption of nondogmaticity of the BBA $m(\cdot)$ is necessary for Smets canonical decomposition ${ }^{3}$, but it is not essential for our PCR5-based canonical decomposition because it also works with a dogmatic BBA as discussed in section 6 .

The belief function canonical decomposition problem (BF-CDP) can be expressed as follows:

Given a nondogmatic BBA $m(\cdot)$ as in (4) and a chosen rule of combination, find the two following simple proper BBAs $m_{p}$ and $m_{c}$ of the form

$$
\begin{aligned}
& m_{p}(A)=x, \quad m_{p}(A \cup \bar{A})=1-x \\
& m_{c}(\bar{A})=y, \quad m_{c}(A \cup \bar{A})=1-y
\end{aligned}
$$

with $(x, y) \in[0,1] \times[0,1]$, such that $m=$ Fusion $\left(m_{p}, m_{c}\right)$, for a chosen rule of combination denoted Fusion $(\cdot, \cdot)$.

$m_{p}(\cdot)$ is called the pro- $B B A$ (or pro-evidence) of $A$, and $m_{c}(\cdot)$ the contra-BBA (or contra-evidence) of $A$. In the section 5 we prove that this decomposition is always possible and unique and we call it the (PCR5-based) canonical decomposition of the BBA $m(\cdot)$. The BBA $m_{p}(\cdot)$ is interpreted as a source of evidence providing uncertain evidence in favor of $A$, whereas $m_{c}(\cdot)$ is interpreted as a source of evidence providing uncertain evidence against $A$. The BBA $m(\cdot)$ can be interpreted as the result of the PCR5 fusion of these two (pros and cons) aspects of evidence about $A$.

It is worth noting that this BF-CDP must not be confused with canonical decomposition problem addressed by Smets in ${ }^{3}$ in his TBM framework, which is based on conjunctive rule of combination and which involves, in general, improper BBAs, called generalized simple BBA (GSBBA) in Smets terminology.

\footnotetext{
${ }^{5}$ which means that $m(\emptyset)=0$.
} 


\subsection{Impossibility of decompositions by some well-known rules}

Here we analyze briefly the impossibility of a canonical decomposition for some wellknown rules of combination.

\subsubsection{Conjunctive rule}

We consider $x>1$ and $y>1$ so that the two BBAs are really informative (otherwise they become vacuous and useless from decision-maing standpoint). In this case we always have a conflict between $m_{p}(\cdot)$ and $m_{c}(\cdot)$ resulting of the conjunctive rule of combination. That is

$$
m_{\text {conj }}(\emptyset)=m_{p}(A) m_{c}(\bar{A})=x \cdot y>0
$$

Hence $m_{\text {conj }}(\emptyset) \neq 0$ is incompatible with the constraint $m(\emptyset)=0$. Therefore, the canonical decomposition of the BBA $m(\cdot)$ expressed as the conjunctive fusion of pros and cons BBAs $m_{p}(\cdot)$ and $m_{c}(\cdot)$ is impossible to get in general ${ }^{6}$, but in the very degenerate cases where $a=0$, or $b=0$, or $a=0$ and $b=0$ which would involve vacuous BBAs in the decomposition and of course will be useless.

\subsubsection{Disjunctive rule}

If we consider the disjunctive rule of combination of $m_{p}(\cdot)$ and $m_{c}(\cdot)$ we will always obtain the vacuous BBA because $m_{p}(A) m_{c}(\bar{A}), m_{p}(A) m_{c}(A \cup \bar{A}), m_{p}(A \cup \bar{A}) m_{c}(\bar{A})$ and $m_{p}(A \cup \bar{A}) m_{c}(A \cup \bar{A})$ will all be committed to the uncertainty $A \cup \bar{A}$. Therefore the combination result is nothing but the vacuous belief assignment $m_{v}$, that is $\operatorname{Disj}\left(m_{p}, m_{c}\right)=m_{v}$. In conclusion, we cannot make a decomposition of the BBA $m(\cdot)$ based on the disjunctive rule in general because if $m(\cdot)$ is informative (e.g. not vacuous) one always has $a+b<1$ so that $m(A \cup \bar{A})<1$ whereas the disjunctive rule of $m_{p}(\cdot)$ and $m_{c}(\cdot)$ will always provide $m(A \cup \bar{A})=1$.

\subsubsection{Yager's and Dubois \& Prade rules}

Due to the particular simple form of BBAs $m_{p}(A)$ and $m_{c}(\cdot)$, Yager's rule ${ }^{13}$ and Dubois-Prade rule ${ }^{14}$ coincide. Based on these rules we are searching $x$ and $y$ in $[0,1]$ such that

$$
\begin{aligned}
m(A) & =a=x(1-y) \\
m(\bar{A}) & =b=(1-x) y \\
m(A \cup \bar{A}) & =1-a-b=(1-x)(1-y)+x y
\end{aligned}
$$

Because the third equation is dependent of the two first, we have only to solve the following system of equations $x-x y=a$ and $y-x y=b$. Assuming ${ }^{7} y<1$, one gets from the first equation $x=\frac{a}{1-y}$. By replacing $x$ by its expression in the

\footnotetext{
${ }^{6}$ that is for any $a$ and $b$ values of mass of focal elements $A$ and $\bar{A}$ of the BBA $m \cdot$.).

${ }^{7}$ taking $y=1$ would means that $x(1-y)=0$ but $m(A)=a$ with $a \neq 0$ in general, so that the choice of $y=1$ is not possible.
} 
second equation $y-x y=b$ we have to find $y$ in $[0,1)$ such that (after basic algebraic simplifications)

$$
y^{2}+(a-b-1) y+b=0
$$

This second order equation admits one or two real solutions $y_{1}$ and $y_{2}$ if and only if the discriminant is null or positive respectively, that is if $(a-b-1)^{2}-4 b \geq 0$. However this discriminant can become negative depending on the values of $a$ and $b$. For instance, for $a=0.4$ and $b=0.5$, we have $(a-b-1)^{2}-4 b=-0.79$ which means that there is no real solution for the equation $y^{2}-1.1 \cdot y+0.5=0$. Therefore, in general, the canonical decomposition of the BBA $m(\cdot)$ cannot be accomplished from Yager's and Dubois \& Prade rules of combination.

\subsubsection{Averaging rule}

Suppose we combine $m_{p}(\cdot)$ and $m_{c}(\cdot)$ with the averaging rule. Then we are searching $x$ and $y$ in $[0,1]$ such that

$$
\begin{aligned}
m(A) & =a=(x+0) / 2 \\
m(\bar{A}) & =b=(0+y) / 2 \\
m(A \cup \bar{A}) & =1-a-b=((1-x)+(1-y)) / 2
\end{aligned}
$$

This means that $x=2 a$ and $y=2 b$ with $x$ and $y$ in $[0,1]$. So, if $a>0.5$ or $b>0.5$ the canonical decomposition is impossible to make with the averaging rule of combination. Therefore, in general, the averaging rule is not able to provide a canonical decomposition of the BBA $m(\cdot)$.

\section{DECOMPOSITION BASED ON DEMPSTER'S RULE}

Let consider a nondogmatic BBA $m(A)=a, m(\bar{A})=b$ and $m(A \cup \bar{A})=1-a-b$ with $0 \leq a, b \leq 1$ and $1-a-b>0$, and let's see if a decomposition of $(\cdot)$ is possible based on Dempster's rule of combination ${ }^{1}$. For this, we are searching $x$ and $y$ in $[0,1]$ such that $x y \neq 1$ and

$$
\begin{aligned}
& m(A)=a=\frac{x(1-y)}{1-x y} \\
& m(\bar{A})=b=\frac{y(1-x)}{1-x y} \\
& m(A \cup \bar{A})=1-a-b=\frac{(1-x)(1-y)}{1-x y}
\end{aligned}
$$

Because the third equality is redundant with the two first, we just have to solve the system of two equations expressed as

$$
\begin{aligned}
& (1-x y) a=x(1-y) \\
& (1-x y) b=y(1-x)
\end{aligned}
$$


That is, one should have

$$
\begin{aligned}
& x-x y+a x y=a \\
& y-x y+b x y=b
\end{aligned}
$$

with the constraints $0<x<1$ and $0<y<1$. So one must have

$$
x=\frac{a}{1-y+a y}, \quad y \neq \frac{1}{1-a}
$$

and solve the equation $y-x y+b x y=b$ with $x$ expressed as function of $y$ as above. We get the equation for $a \neq 1$

$$
(a-1) y^{2}+(1+b-a) y-b=0
$$

whose solutions have the form

$$
y=\frac{-(1+b-a) \pm \sqrt{\Delta}}{2(a-1)}
$$

where the discriminant $\Delta$ is given by

$$
\begin{aligned}
\Delta & =(1+b-a)^{2}-4(1-a) b=1+b^{2}+a^{2}+2 b-2 a-2 a b+4 a b-4 b \\
& =a^{2}+b^{2}+1-2 b+2 a b-2 a=(a+b-1)^{2}=(1-a-b)^{2}
\end{aligned}
$$

One sees that $\Delta$ is strictly positive because $a+b<1$ ( $m$ being a nondogmatic BBA). So, there exist two real solutions $y_{1}$ and $y_{2}$ of (23) of the form

$$
\begin{gathered}
y_{1}=\frac{-(1+b-a)+\sqrt{\Delta}}{2(a-1)}=\frac{b}{1-a} \\
y_{2}=\frac{-(1+b-a)-\sqrt{\Delta}}{2(a-1)}=\frac{1-a}{1-a}=1
\end{gathered}
$$

For the case $a \neq 1$, the second "solution" $y_{2}=1$ implies $x=\frac{a}{1-y_{2}+a y_{2}}=\frac{a}{a}=1$ which is not an acceptable solution ${ }^{8}$ because one must have $x y \neq 1$. The solution $(x, y)$ of the decomposition problem for $a \neq 1$ is actually given by the first solution $y_{1}$, that is

$$
\begin{aligned}
& y=y_{1}=\frac{b}{1-a} \in[0,1) \\
& x=\frac{a}{1-y+a y}=\frac{a}{1-b} \in[0,1)
\end{aligned}
$$

The case $a=1$ corresponding to the dogmatic BBA given by $m(A)=a=1$, $m(\bar{A})=b=0, m(A \cup \bar{A})=1-a-b=0$ is analyzed in details in Section 6 - See lemma right after Theorem 4 .

\footnotetext{
${ }^{8}$ otherwise the denominators of Eqs. (15)-(17) will be equal to zero.
} 
In summary, the unique solution of decomposition of a nondogmatic BBA with $0<a<1,0<b<1$ and $a+b<1$ using Dempster's rule is $x=\frac{a}{1-b}$ and $y=\frac{b}{1-a}$.

Example 1: Consider $m(A)=a=0.6, m(\bar{A})=b=0.2$ and $m(A \cup \bar{A})=1-a-b=$ 0.2 . The solution $(x, y)$ of the decomposition of $m(\cdot)$ based on Dempster's rule is $x=$ $\frac{a}{1-b}=\frac{0.6}{1-0.2}=0.75$ and $y=\frac{b}{1-a}=\frac{0.2}{1-0.6}=0.5$. Therefore, $m_{p}(A)=x=0.75$, $m_{p}(A \cup \bar{A})=1-x=0.25$ and $m_{c}(\bar{A})=y=0.5, m_{c}(A \cup \bar{A})=1-y=0.5$. It can be verified that $m_{p} \oplus m_{c}=m$, where $\oplus$ represents symbolically Dempster's rule of combination ${ }^{1}$.

\section{DECOMPOSITION BASED ON PCR5 RULE}

In this section we prove that the decomposition of a dichotomous nondogmatic BBA $m(\cdot)$ based on PCR5 rule of combination is always possible and unique. Suppose we combine $m_{p}(\cdot)$ and $m_{c}(\cdot)$ with the PCR5 rule of combination. Then we are searching $(x, y) \in[0,1]^{2}$ satisfying

$$
\begin{aligned}
& m(A)=a=x(1-y)+\frac{x^{2} y}{x+y}=\frac{x^{2}+x y-x y^{2}}{x+y} \\
& m(\bar{A})=b=(1-x) y+\frac{x y^{2}}{x+y}=\frac{y^{2}+x y-x^{2} y}{x+y} \\
& m(A \cup \bar{A})=1-a-b=1-x-y+x y
\end{aligned}
$$

under the constraints $(a, b) \in[0,1]^{2}$, and $0<a+b<1$.

The equations (29) and (30) can be rewritten as

$$
\begin{aligned}
& x-\frac{x y^{2}}{x+y}=a \\
& y-\frac{x^{2} y}{x+y}=b
\end{aligned}
$$

from which (31) is redundant because (29) + (30) gives

$$
x+y-x y=a+b
$$

Therefore $(1-x)(1-y)=1-(a+b)$ and that is why the constraint $a+b \leq 1$ is necessary ${ }^{9}$ for the existence of the solution $(x, y)$.

With $x$ and $y$ in $[0,1]$ the solutions of (32) and (33) verify

$$
\begin{aligned}
& x \geq a \\
& y \geq b
\end{aligned}
$$

Moreover, the equality (34) implies

$$
\begin{aligned}
& x(1-y)=a+b-y \quad \Rightarrow \quad y \leq a+b \\
& y(1-x)=a+b-x \quad \Rightarrow \quad x \leq a+b
\end{aligned}
$$

\footnotetext{
${ }^{9}$ In fact we use the constraint $a+b<1$ because in this section we consider only nondogmatic BBA. The canonical decomposition of a dichotomous dogmatic BBA will be analyzed in the section 6 .
} 
For $x \neq 1$, from (34) one gets $y=\frac{a+b-x}{1-x}$ and from (32) one has

$$
x^{2}+x y-x y^{2}=a x+a y
$$

Putting this expression of $y$ in (39), yields the equation

$$
x^{2}+(x-a) \frac{a+b-x}{1-x}-x\left(\frac{a+b-x}{1-x}\right)^{2}-a x=0
$$

which can be expressed after elementary algebraic calculation as

$$
x^{4}+(-a-2) x^{3}+(2 a+b) x^{2}+\left(a+b-a b-b^{2}\right) x+\left(-a^{2}-a b\right)=0
$$

This equation of degree 4 has at most four real solutions. We have to take only the solution $x$ from the open interval $(0,1)$ and $y=(a+b-x) /(1-x)$ with $y \in[0,1]$.

The general expression of the solutions of this quartic equation ${ }^{15}$ is very complicate to obtain analytically even with modern symbolic computing systems like Maple ${ }^{\mathrm{TM}}$, or Mathematica $^{\mathrm{TM}}$, but the solutions can be easily calculated numerically by these computing systems, and even with Matlab ${ }^{\mathrm{TM}}$ system (thanks to the fsolve command) as soon as the numerical values are committed to $a$ and to $b$. Another method to make the decomposition consists to solve numerically the system of equations $\frac{x^{2}+x y-x y^{2}}{x+y}=a$ and $\frac{y^{2}+x y-x^{2} y}{x+y}=b$ for numerical values committed to $a$ and $b$ thanks to Mathematica ${ }^{\mathrm{TM}}$, Maple $^{\mathrm{TM}}$, or Matlab ${ }^{\mathrm{TM}}$ computing systems for instance. Of course the solutions provided by the two methods are the same.

Example 2: Let consider $m(A)=0.6, m(\bar{A})=0.3$ and $m(A \cup \bar{A})=0.1$, therefore $a=0.6$ and $b=0.3$. The quartic equation (41) becomes

$$
x^{4}-2.6 x^{3}+1.5 x^{2}+0.63 x-0.54=0
$$

The four solutions of this quartic equation provided by the computing system ${ }^{10}$ are approximately

$$
\begin{aligned}
& x_{1} \approx 0.7774780438 \\
& x_{2} \approx 0.9297589637 \\
& x_{3} \approx 1.419151582 \\
& x_{4} \approx-0.5263885898
\end{aligned}
$$

which are shown on the graph of figure 1 obtained easily from Desmos online tool ${ }^{11}$.

Clearly $x_{3}$ and $x_{4}$ are not acceptable solutions because they don't belong to [0,1]. If we take $x_{1} \approx 0.7774780438$ then will get $y_{1}=\left(a+b-x_{1}\right) /\left(1-x_{1}\right)=(0.9-$ $\left.x_{1}\right) /\left(1-x_{1}\right) \approx 0.5506061437$, so the pair $\left(x_{1}, y_{1}\right) \in[0,1]^{2}$ is a solution of the decomposition problem of the BBA $m(\cdot)$. If we take $x_{2} \approx 0.9297589637$ then will get $y_{2}=\left(a+b-x_{2}\right) /\left(1-x_{2}\right)=\left(0.9-x_{2}\right) /\left(1-x_{2}\right) \approx-0.4236692006$, so we see that

\footnotetext{
${ }^{10}$ We did also obtain the same solutions with Maple ${ }^{\mathrm{TM}}$, and also with Matlab ${ }^{\mathrm{TM}}$.

${ }^{11}$ https://www. desmos.com/calculator
} 


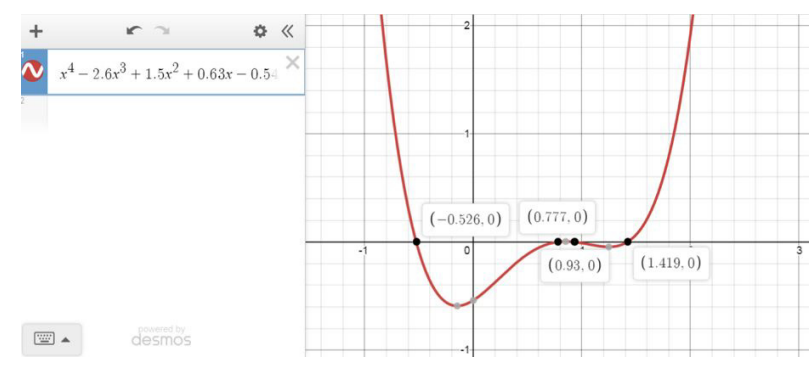

Figure 1: Plot of the quartic function.

$y_{2} \notin[0,1]$ and therefore the pair $\left(x_{2}, y_{2}\right)$ cannot be a solution of the decomposition problem of the BBA $m(\cdot)$. Therefore the canonical masses $m_{p}(\cdot)$ and $m_{c}(\cdot)$ are given by

$$
\begin{aligned}
& m_{p}(A) \approx 0.7774780438, \quad m_{p}(A \cup \bar{A}) \approx 0.2225219562 \\
& m_{c}(\bar{A}) \approx 0.5506061437, \quad m_{c}(A \cup \bar{A}) \approx 0.4493938563
\end{aligned}
$$

It can be verified that the PCR5 combination of the BBAs $m_{p}$ and $m_{c}$, denoted $P C R 5\left(m_{p}, m_{c}\right)$, is equal to the BBA $m(\cdot)$. The following important theorem holds.

Theorem 1: Consider a dichotomous FoD $\Theta=\{A, \bar{A}\}$ with $A \neq \Theta$ and $A \neq \emptyset$ and a nondogmatic BBA $m(\cdot): 2^{\Theta} \rightarrow[0,1]$ defined on $\Theta$ by $m(A)=a, m(\bar{A})=b$, and $m(A \cup \bar{A})=1-a-b$, where $a, b \in[0,1]$ and $a+b<1$. Then the BBA $m(\cdot)$ has a unique canonical decomposition using PCR5 rule of combination of the form $m=\operatorname{PCR} 5\left(m_{\underline{p}}, m_{c}\right)$ with pro-evidence $m_{p}(A)=x, m_{p}(A \cup \bar{A})=1-x$ and contra-evidence $m_{c}(\bar{A})=y, m_{c}(A \cup \bar{A})=1-y$, where $x, y \in[0,1]$.

Proof: Based on (29)-(30), we have to prove that the following system $S_{a, b}$ of equations always admits one and only one solution $(x, y) \in[0,1] \times[0,1]$

$$
S_{a, b}:\left\{\begin{array}{l}
h(x, y)=a \\
h(y, x)=b
\end{array}\right.
$$

with $h(x, y)=\frac{x^{2}+x y-x y^{2}}{x+y}=x-\frac{x y^{2}}{x+y}$. The $h$ function can be prolonged in $(0,0)$ by continuity by setting $h(0,0)=0$.

One has to prove the existence of a unique $x \in[a, a+b] \subset[0,1]$ and $y \in[b, a+b] \subset$ $[0,1]$ solutions of $S_{a, b}$, or equivalently solutions of $y=\frac{a+b-x}{1-x}$ and of (41) $P(x)=0$ with

$$
P(x) \triangleq x^{4}+(-a-2) x^{3}+(2 a+b) x^{2}+(a+b)(1-b) x-a(a+b)
$$


Because $^{12} \lim _{x \rightarrow-\infty} P(x)=+\infty$ and $^{13} P(a)<0$, there exists $x_{1} \in(-\infty, a)$ such that $P\left(x_{1}\right)=0$. The solution $x_{1}$ is not acceptable because $x_{1} \notin[a, a+b]$. Because $\mathrm{B}^{14}$ $P(1)<0$ and $\lim _{x \rightarrow+\infty} P(x)=+\infty$, there exists also $x_{4} \in(1,+\infty)$ such that $P\left(x_{4}\right)=$ 0 . The solution $x_{4}$ is not acceptable because $x_{4} \notin[a, a+b]$. For $a+b \neq 1$, one has $^{15} P(a+b)>0$ and $P(1)<0$. Therefore there exists $x_{3} \in(a+b, 1)$ such that $P\left(x_{3}\right)=0$ but this solution $x_{3}$ is also not acceptable because $x_{3} \notin[a, a+b]$. Because $P(a)<0$ and $P(a+b)>0$ there exists $x_{2} \in[a, a+b]$ such that $P\left(x_{2}\right)=0$ which is the only satisfactory solution. The value $y_{2}$ is given by $y_{2}=\frac{a+b-x_{2}}{1-x_{2}}$, and one has $y_{2}>0$ because $x_{2}<a+b$ and $y_{2}<1$ because $a+b<1$. Moreover, from (33), $y_{2}-b=\frac{x_{2}^{2} y_{2}}{x_{2}+y_{2}}$ which is always positive, therefore $y_{2}>b$, and from (34) $y_{2}-(a+b)=x_{2}\left(y_{2}-1\right)$ which is always negative, therefore $y_{2}<a+b$. This completes the proof of the theorem 1.

\section{PARTICULAR CASES OF DECOMPOSITIONS}

Here we examine the canonical decomposition of particular cases, including dogmatic BBA.

\subsection{Dogmatic BBA: $a+b=1$}

Theorem 2: Any dogmatic BBA defined by $m(A)=a$ and $m(\bar{A})=b$, where $a, b \in$ $[0,1]$ and $a+b=1$, has a canonical decomposition using PCR5 rule of combination of the form $m=P C R 5\left(m_{p}, m_{c}\right)$ with $m_{p}(A)=x, m_{p}(A \cup \bar{A})=1-x$ and $m_{c}(\bar{A})=y$, $m_{c}(A \cup \bar{A})=1-y$ where $x, y \in[0,1]$.

Proof: Any solution of $S_{a, b}$ verifies

$$
\begin{aligned}
& x-a=\frac{x y^{2}}{x+y} \\
& y-b=\frac{x^{2} y}{x+y}
\end{aligned}
$$

and therefore from $(45)+(46)$ one has

$$
(x-y)-(a-b)=\frac{x y(y-x)}{x+y}
$$

which can be rewritten as

$$
(x-y)\left[1+\frac{x y}{x+y}\right]=(a-b)
$$

\footnotetext{
${ }^{12} P(x)$ being polynomial, it is continuous and if $P(c) P(d)<0$ there exist at least one solution between $[c, d]$. Therefore, we are not sure a priori there is only one solution between $[c, d]$. In our case, the signs of $P(x)$ for $x=-\infty, a, a+b, 1,+\infty$ are respectively,,,+-+- and + . But because one has 4 intervals, into each interval it is not possible to have more than one solution (because otherwise will get 5 or more solutions, while this equation has only up to 4 real solutions). Therefore in each interval there exists only one real solution.

${ }^{13}$ because $P(a)=a^{2} b-a b(a+b)=-a b^{2}$.

${ }^{14}$ because $P(1)=-1+a+b+(a+b)(1-b-a)=-(a+b-1)^{2}$.

${ }^{15}$ because from (40), $P(a+b) /(1-a-b)^{2}=(a+b)^{2}-a(a+b) \Rightarrow P(a+b)=b(a+b)(1-a-b)^{2}>0$.
} 
This means that differences $(x-y)$ and $(a-b)$ have the same sign. Moreover from (34) with $a+b=1$ one has $x+y-x y=1$, or equivalently $(1-x)(1-y)=0$ which is satisfied if $x=1$, or if $y=1$ or both equal one. We must distinguish three cases as follows:

- If $a<b$ then $^{16} x<y$ therefore $y=1$ and $h(x, 1)=a$. Solving $h(x, 1)=a$ is equivalent to solve $x^{2}-a x-a=0$ which admits only one positive solution $x \in[a, a+b=1]$ given by $x=\frac{a+\sqrt{a^{2}+4 a}}{2}$. Note if $a+b=1$ and $a<b$, then necessarily $a<0.5$.

- If $a>b$ then $x>y$ therefore $x=1$ and $h(1, y)=b$. Solving $h(1, y)=b$ is equivalent to solve $y^{2}-b y-b=0$ which admits only one positive solution $y \in[b, a+b=1]$ given by $y=\frac{b+\sqrt{b^{2}+4 b}}{2}$. Note if $a+b=1$ and $a>b$, then necessarily $b<0.5$.

- If $a=b$ and $a+b=1$ then $a=b=0.5$ and $x=y=1$.

So we have proved that a decomposition based on PCR5 always exists and it is unique also for any dogmatic dichotomous BBA. Therefore this decomposition of dogmatic dichotomous BBA is canonical, which completes the proof of theorem 2.

Theorem 3: Any dogmatic BBA $m(A)=a, m(\bar{A})=b$ with $a+b=1$ and $0<a<1$ is not decomposable from Yager's rule and Dubois-Prade rule of combination.

Proof: We have the following system of equations to solve

$$
\begin{aligned}
& x-x y=a \\
& y-x y=b
\end{aligned}
$$

From (49) and (50), we get $a-b=x-x y-(y-x y)=x-y$, so $y=x-a+b$. After replacing this expression of $y$ into (49) and algebraic manipulations, we have to solve

$$
x^{2}-2 a x+a=0
$$

whose solutions are of the form

$$
x=a \pm \sqrt{a(a-1)}
$$

For $0<a<1$ the system has no real solutions because $a(a-1)<0$, which completes the proof of Theorem 3 .

Theorem 4: Any dogmatic BBA $m(A)=a, m(\bar{A})=b$ with $a+b=1$ is not decomposable from Dempster's rule of combination for the case when $(a, b) \neq(1,0)$ and $(a, b) \neq(0,1)$.

Proof: We have the following system of equations to solve with $0 \leq x, y \leq 1$ and $1-x y \neq 0$

$$
\begin{aligned}
& \frac{x-x y}{1-x y}=a \\
& \frac{y-x y}{1-x y}=b
\end{aligned}
$$

\footnotetext{
${ }^{16}$ because $(x-y)$ and $(a-b)$ have the same sign.
} 
After adding the two equations (51) and (52) and because $a+b=1$, we obtain $\frac{x-x y+y-x y}{1-x y}=a+b=1$, whence $x+y-2 x y=1-x y$, or $x+y-x y=1$, or $x+y(1-x)=1$, or $y(1-x)=1-x$, or $=\frac{1-x}{1-x}=1$ when $x \neq 1$. From (52), one should have $\frac{y-x y}{1-x y}=b$ with $y=1$, that is $\frac{1-x \cdot 1}{1-x \cdot 1}=b$, or $1=b$ which is false because if $0<a<1$ then $b=1-a \neq 1$. This completes the proof of theorem 4 .

Lemma: The dogmatic BBAs $m(A)=1, m(\bar{A})=0$ (case $(a, b)=(1,0)$ ), or $m(A)=$ $0, m(\bar{A})=1$ (case $(a, b)=(0,1))$ have infinitely many decompositions based on Dempster's rule of combination.

Proof: For the case $(a, b)=(1,0)$ one has to solve with $0 \leq x, y \leq 1$ and $1-x y \neq 0$ the system of equations

$$
\frac{x-x y}{1-x y}=1 \quad \text { and } \quad \frac{y-x y}{1-x y}=0
$$

This system is satisfied for $x=1$ and $y \in[0,1)$, that is any value in $[0,1)$ can be chosen for $y$.

For the case $(a, b)=(0,1)$ one has to solve with $0 \leq x, y \leq 1$ and $1-x y \neq 0$ the system of equations

$$
\frac{x-x y}{1-x y}=0 \quad \text { and } \quad \frac{y-x y}{1-x y}=1
$$

This system is satisfied for $y=1$ and $x \in[0,1)$, that is any value in $[0,1)$ can be chosen for $x$. Therefore one sees that for the case $(a, b)=(1,0)$ and the case $(a, b)=(0,1)$ there is no unique decomposition of these BBAs from Dempster's rule of combination, which completes the proof of the lemma.

\subsection{Case when $a=0$ and $b=0$ (i.e. $m$ is the vacuous BBA)}

This is the most degenerate case where the BBA $m(\cdot)$ corresponds to the vacuous BBA. For averaging rule, conjunctive rule, Yager's, Dubois-Prade's, Dempster's and PCR5 rules one has $x=0$ and $y=0$ (conflict between canonical masses is zero). In fact the vacuous BBA $m(\cdot)$ can always be interpreted as the fusion of $m_{p}$ and $m_{c}$, where $m_{p}$ and $m_{c}$ are also vacuous BBAs. This degenerate case has no particular interest in practice but to model the total ignorant state of knowledge.

\subsection{Case when $a=0$, or $b=0$}

In the case $a=0$ and $0<b \leq 1$, then for conjunctive rule, Yager's, Dubois-Prade's, Dempster's and PCR5 rules one has $x=0$ and $y=b$ (conflict between canonical masses is zero) and $m(\cdot)$ corresponds to the fusion of vacuous pro-evidence $m_{p}=m_{v}$ with the contra-evidence $m_{c}=m$. In the case $0<a \leq 1$ and $b=0$, then for conjunctive rule, Yager's, Dubois-Prade's, Dempster's and PCR5 rules one has $x=a$ and $y=0$ (conflict between canonical masses is zero) and $m(\cdot)$ corresponds to the fusion of the pro-evidence $m_{p}=m$ with the vacuous contra-evidence $m_{c}=m_{v}$. These cases have no particular interest because they can be seen just as the combination of pros (or cons) BBA with the vacuous BBA 


\subsection{Case when $a=b \in(0,0.5)$}

Theorem 5: In the case $a=b \in(0,0.5)$, the BBA $m(A)=m(\bar{A})=a$ and $m(A \cup$ $\bar{A})=1-2 a$ can be canonically decomposed from PCR5 rule with the BBAs $m_{p}(A)=$ $1-\sqrt{1-2 a}, m_{p}(A \cup \bar{A})=\sqrt{1-2 a}$ and $m_{c}(\bar{A})=1-\sqrt{1-2 a}, m_{c}(A \cup \bar{A})=$ $\sqrt{1-2 a}$.

Proof: From (29) and (30), one has $\frac{x^{2}+x y-x y^{2}}{x+y}=a$ and one has also in this case $\frac{y^{2}+x y-x^{2} y}{x+y}=b=a$. Therefore $x^{2}+x y-x y^{2}=y^{2}+x y-x^{2} y$, or $x^{2}-x y^{2}-y^{2}+x^{2} y=$ 0 , or $(x-y)(x+y+x y)=0 . x \geq 0$ and $y \geq 0$ because they represent the masses. Therefore $x+y+x y \geq 0$. The sum $x+y+x y=0$ if and only if $x=y=0$, but this produces the degenerate case, which is corresponding to $a=b=0$ (i.e. the vacuous BBA). Yet, in our theorem's hypothesis we assumed $a, b \in(0,0.5)$, so $a>0$, and $b>0$. Therefore $x+y+x y>0$. Hence $x=y$. Therefore the canonical BBAs must be of the form $m_{p}(A)=x, m_{p}(A \cup \bar{A})=1-x$ and $m_{c}(\bar{A})=x, m_{c}(A \cup \bar{A})=$ $1-x$. So one must solve the equation ${ }^{17} x-x^{2}+\frac{x^{2}}{2}=m(A)=a$, or equivalently $\frac{1}{2} x^{2}-x+a=0$, whose solutions are $x_{1}=1+\sqrt{1-2 a}$, and $x_{2}=1-\sqrt{1-2 a}$. For $0<a<0.5$, the solution $x_{1}>1$ is not admissible because $x_{1} \notin[0,1]$. The solution $x_{2}$ is acceptable because if $0<a<0.5$, then $0<2 a<1$, or $-1<-1+2 a<0$, or (by multiplying by -1 the inequalities) $1>1-2 a>0$, or $0<1-2 a<1$, or $\sqrt{0}<\sqrt{1-2 a}<\sqrt{1}$, or $0>-\sqrt{1-2 a}>-1$, or $1>1-\sqrt{1-2 a}>0$ hence $x_{2} \in(0,1)$. This completes the proof of the theorem 5 .

\section{EXAMPLES}

We give in Tables I-IX some numerical examples of PCR5-based canonical decompositions of BBA $m(\cdot)$ for different sampled values of $a$ and $b$ for convenience. These numerical examples may be useful for researchers working with belief functions and interested by this new type of decomposition in their own examples. The values have been approximated at the 10th digit. Figures 2 and 3 show the shapes of the proevidence $x=f(a, b)$ and the contra-evidence $y=g(a, b)$ surfaces proving graphically the existence of canonical decomposition based on PCR5 at the sampling rate of 0.025 . The values $(a, b)$ for which $a+b>1$ are not acceptable and $f(a, b)$ and $g(a, b)$ have been set to zero in the figures.

\footnotetext{
${ }^{17}$ In fact, we have also the second equation $x-x^{2}+\frac{x^{2}}{2}=m(\bar{A})=b=a$ to solve which is the same as the first one.
} 


\begin{tabular}{|c|c|c|}
\hline$(a, b)$ & $x$ & $y$ \\
\hline$(0.1,0.1)$ & 0.1055728059 & 0.1055728059 \\
$(0.1,0.2)$ & 0.1155063468 & 0.2085867463 \\
$(0.1,0.3)$ & 0.1283308324 & 0.3116654549 \\
$(0.1,0.4)$ & 0.1445620975 & 0.4155040377 \\
$(0.1,0.5)$ & 0.1653570911 & 0.5207531320 \\
$(0.1,0.6)$ & 0.1926613985 & 0.6284087006 \\
$(0.1,0.7)$ & 0.2298437881 & 0.7403124237 \\
$(0.1,0.8)$ & 0.2834628414 & 0.8604398965 \\
$(0.1,0.9)$ & 0.3701562119 & 1 \\
\hline
\end{tabular}

Table I: Decomposition of BBA when $m(A)=0.1$.

\begin{tabular}{|c|c|c|}
\hline$(a, b)$ & $x$ & $y$ \\
\hline$(0.2,0.1)$ & 0.2085867463 & 0.1155063468 \\
$(0.2,0.2)$ & 0.2254033308 & 0.2254033308 \\
$(0.2,0.3)$ & 0.2477759456 & 0.3353044255 \\
$(0.2,0.4)$ & 0.2763932022 & 0.4472135955 \\
$(0.2,0.5)$ & 0.3133633342 & 0.5630877072 \\
$(0.2,0.6)$ & 0.3628331876 & 0.6861104563 \\
$(0.2,0.7)$ & 0.4339764332 & 0.8233289109 \\
$(0.2,0.8)$ & 0.5582575695 & 1 \\
\hline
\end{tabular}

Table II: Decomposition of BBA when $m(A)=0.2$.

\begin{tabular}{|c|c|c|}
\hline$(a, b)$ & $x$ & $y$ \\
\hline$(0.3,0.1)$ & 0.3116654549 & 0.1283308324 \\
$(0.3,0.2)$ & 0.3353044255 & 0.2477759456 \\
$(0.3,0.3)$ & 0.3675444680 & 0.3675444680 \\
$(0.3,0.4)$ & 0.4098895428 & 0.4916206002 \\
$(0.3,0.5)$ & 0.4669657064 & 0.6247896197 \\
$(0.3,0.6)$ & 0.5506061437 & 0.7774780438 \\
$(0.3,0.7)$ & 0.7178908346 & 1 \\
\hline
\end{tabular}

Table III: Decomposition of BBA when $m(A)=0.3$. 


\begin{tabular}{|c|c|c|}
\hline$(a, b)$ & $x$ & $y$ \\
\hline$(0.4,0.1)$ & 0.4155040377 & 0.1445620975 \\
$(0.4,0.2)$ & 0.4472135955 & 0.2763932022 \\
$(0.4,0.3)$ & 0.4916206002 & 0.4098895428 \\
$(0.4,0.4)$ & 0.5527864045 & 0.5527864045 \\
$(0.4,0.5)$ & 0.6442577571 & 0.7188975951 \\
$(0.4,0.6)$ & 0.8633249581 & 1 \\
\hline
\end{tabular}

Table IV: Decomposition of BBA when $m(A)=0.4$.

\begin{tabular}{|c|c|c|}
\hline$(a, b)$ & $x$ & $y$ \\
\hline$(0.5,0.1)$ & 0.5207531320 & 0.1653570911 \\
$(0.5,0.2)$ & 0.5630877072 & 0.3133633342 \\
$(0.5,0.3)$ & 0.6247896197 & 0.4669657064 \\
$(0.5,0.4)$ & 0.7188975951 & 0.6442577571 \\
$(0.5,0.5)$ & 1 & 1 \\
\hline
\end{tabular}

Table V: Decomposition of BBA when $m(A)=0.5$.

\begin{tabular}{|c|c|c|}
\hline$(a, b)$ & $x$ & $y$ \\
\hline$(0.6,0.1)$ & 0.6284087006 & 0.1926613985 \\
$(0.6,0.2)$ & 0.6861104563 & 0.3628331876 \\
$(0.6,0.3)$ & 0.7774780438 & 0.5506061437 \\
$(0.6,0.4)$ & 1 & 0.8633249581 \\
\hline
\end{tabular}

Table VI: Decomposition of BBA when $m(A)=0.6$.

\begin{tabular}{|c|c|c|}
\hline$(a, b)$ & $x$ & $y$ \\
\hline$(0.7,0.1)$ & 0.7403124237 & 0.2298437881 \\
$(0.7,0.2)$ & 0.8233289109 & 0.4339764332 \\
$(0.7,0.3)$ & 1 & 0.7178908346 \\
\hline
\end{tabular}

Table VII: Decomposition of BBA when $m(A)=0.7$.

\begin{tabular}{|c|c|c|}
\hline$(a, b)$ & $x$ & $y$ \\
\hline$(0.8,0.1)$ & 0.8604398965 & 0.2834628414 \\
$(0.8,0.2)$ & 1 & 0.5582575695 \\
\hline
\end{tabular}

Table VIII: Decomposition of BBA when $m(A)=0.8$. 


\begin{tabular}{|c|c|c|}
\hline$(a, b)$ & $x$ & $y$ \\
\hline$(0.9,0.1)$ & 1 & 0.3701562119 \\
\hline
\end{tabular}

Table IX: Decomposition of BBA when $m(A)=0.9$.

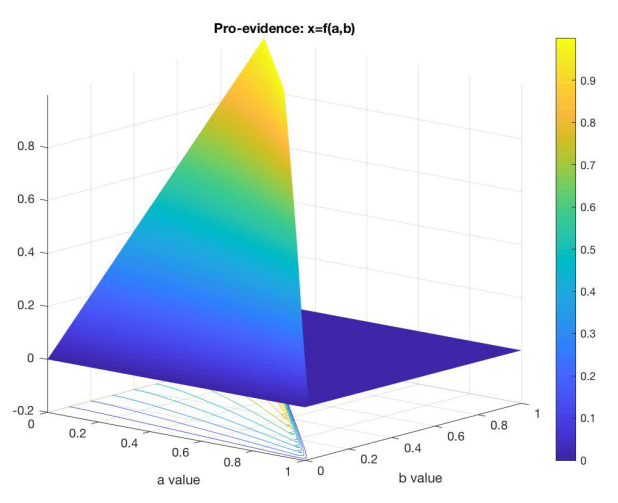

Figure 2: Plot of $x=f(a, b)$ pro-evidence surface.

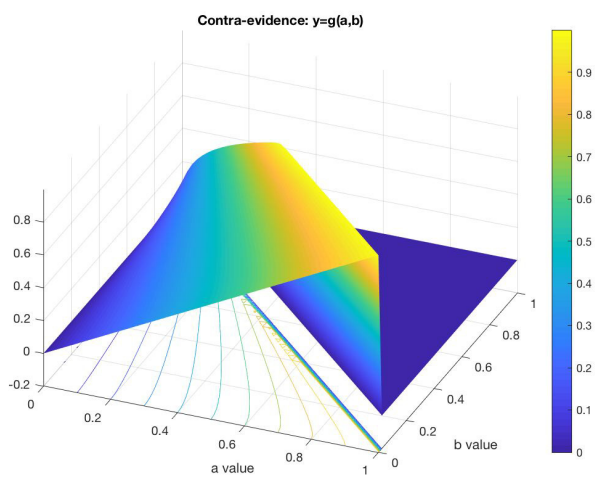

Figure 3: Plot of $y=g(a, b)$ contra-evidence surface. 


\section{INTEREST OF CANONICAL DECOMPOSITION}

The canonical decomposition based on PCR5 offers several practical interests and advantages that are briefly listed here.

1. From the theoretical standpoint, one has proved that the canonical decomposition based on PCR5 rule always exists in all the cases for nondogmatic or dogmatic BBAs contrariwise to other rules of combination that only work in some restrictive cases. Therefore this decomposition is more general and mathematically well justified.

2. This canonical decomposition of any dichotomous BBA $m(\cdot)$ into the pro-evidence $m_{p}(\cdot)$ and the contra-evidence $m_{c}(\cdot)$ allows to define now the notion of internal conflict of a (dichotomous) source of evidence, denoted $K_{\text {int }}(m)$, by

$$
K_{\text {int }}(m) \triangleq m_{p}(A) m_{c}(\bar{A})
$$

where $m_{p}(A)=x$ and $m_{c}(\bar{A})=y$ are the canonical factors of the BBA $m(\cdot)$ based on PCR5 rule of combination. It is worth noting that the BBA $m(\cdot)$ has no internal conflict, if and only if at least one of its factor is the vacuous belief mass, that is if $x=0$ or $y=0$, or both, which makes sense. For instance the BBA $m(A)=0.3$ and $m(A \cup \bar{A})=0.7$ doesn't carry internal conflict because $m_{p}=m$ and $m_{c}=m_{v}$ (the vacuous BBA) so that its internal conflict $K_{\text {int }}(m) \triangleq m_{p}(A) m_{c}(\bar{A})=0.3 \cdot 0=0$. In fact in this example the BBA $m(\cdot)$ carries only uncertain pro-evidence, and vacuous contra-evidence. This internal conflict measure should contribute somehow in the definition of the information content carried by a (dichotomous) source of evidence. This aspect however is not detailed in this paper and is left for future research works. It is clear that the maximum of internal conflict $K_{\text {int }}(m)=1$ is obtained for the dogmatic BBA $m(A)=m(\bar{A})=0.5$ whose canonical decomposition by PCR5 is $m_{p}(A)=1$ and $m_{c}(\bar{A})=1$ which shows the full conflict between the pro-evidence $m_{p}(\cdot)$ and the contra-evidence $m_{c}(\cdot)$ of the source. Of course, there is no internal conflict for the vacuous BBA. More precisely, $K_{\text {int }}\left(m_{v}\right)=0$ because if $a=$ $b=0$ then one has $x=y=0$ calculated from PCR5-based decomposition.

3. This canonical decomposition allows also to define the notion of level of uncertainty $U(m)$ of a dichotomous source of evidence $m(\cdot)$ as the conjunction of the uncertainties of pro and contra evidences, that is

$$
\begin{aligned}
U(m) & \triangleq m_{p}(A \cup \bar{A}) m_{c}(A \cup \bar{A}) \\
& =(1-x)(1-y)=1-x-y+x y \\
& =1-x-y+K_{\text {int }}(m)
\end{aligned}
$$

Because of PCR5-based decomposition one gets (as already shown in (31)) $U(m)=$ $1-a-b$ which always belongs to $[0,1]$. The formula (56) is interesting because it clearly shows the link between the pro-evidence value $x$, the contra-evidence 


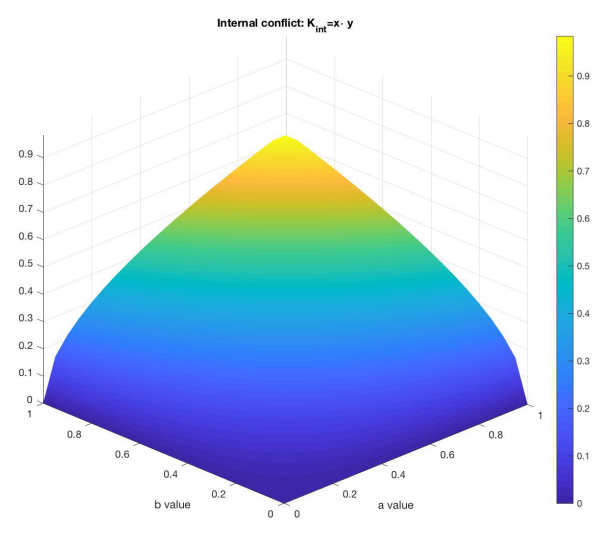

Figure 4: Internal conflict $K_{\text {int }}(m)$.

value $y$ and the internal conflict $K_{\text {int }}(m)=x y$. Clearly, if $x=0$ and $y=0$, then $K_{\text {int }}(m)=0$ and the uncertainty is maximal (i.e. $U(m)=1$ ) because the dichotomous BBA $m$ is the vacuous BBA $m(A \cup \bar{A})=1$. It can be verified that a dichotomous BBA $m$ has no uncertainty $(U(m)=0)$ if and only if $x=1$, or $y=1$, or both which means that $m(\cdot)$ is a Bayesian dichotomous BBA.

4. The canonical decomposition allows also to adjust/revise easily a dichotomous source of evidence (if needed) according the knowledge one has on it. For instance, suppose one knows that the source which provides the BBA $m(\cdot)$ usually over estimates with a reinforcement factor of $\beta_{p}=20 \%$ the belief mass committed to hypothesis $A$ but is always fair (unbiased) when committing its mass to $\bar{A}$. Under this condition, we make the canonical decomposition of $m(\cdot)$ to get $m_{p}(\cdot)$ and $m_{c}(\cdot)$ and we have to discount ${ }^{18}$ the pro-evidence $m_{p}(\cdot)$ with the discounting rate of $\alpha_{p}=1 /\left(1+\beta_{p}\right)$ to get the new unbiased BBA $m_{p}^{\prime}(\cdot)$ and keep the contra-evidence $m_{c}(\cdot)$ unchanged, so that the corrected (unbiased) BBA $m^{\prime}(\cdot)$ will be obtained by the PCR5 combination of $m_{p}^{\prime}(\cdot)$ with $m_{c}(\cdot)$. Of course similar principles can be applied to discount (or reinforce) $m_{c}(\cdot)$ as we prefer (and when necessary) by choosing the adequate discounting (or reinforcing) factors.

5. This canonical decomposition opens the door to new rules of combination for the fusion of $S \geq 2$ (dichotomous) distinct ${ }^{19}$ BBAs $m_{s}(\cdot), s=1,2 \ldots, S$. After making their canonical decompositions to get $S$ pro-evidences $m_{p, s}=$ $\left(m_{p, s}(A), m_{p, s}(\bar{A}), m_{p, s}(A \cup \bar{A})\right)=\left(x_{s}, 0,1-x_{s}\right)$ and $S$ contra-evidences $m_{c, s}=\left(m_{c, s}(A), m_{c, s}(\bar{A}), m_{c, s}(A \cup \bar{A})\right)=\left(0, y_{s}, 1-y_{s}\right)$ for $s=1,2, \ldots, S$, one can for instance combine the $S$ informative non-conflicting pro-evidences

\footnotetext{
${ }^{18}$ We use classical Shafer's discounting method ${ }^{1}$.

${ }^{19}$ i.e. cognitively independent.
} 
$m_{p, s}$ altogether by the conjunctive rule (or any rule one prefers) to get the combined pro-evidence $m_{p}(\cdot)$, and do similarly to combine altogether the non conflicting contra-evidences $m_{c, s}$ to get the combined contra-evidence $m_{c}(\cdot)$. Once $m_{p}(\cdot)$ and $m_{c}(\cdot)$ are calculated, we combine them with PCR5 to get the final resulting BBA. Processing this way will greatly simplify the combination of many dichotomous BBAs. Once the decomposition of each dichotomous BBA is done, we could also consider to apply some importance discounting ${ }^{10}$ with rates $\beta_{s}$ to combine separately the set of BBAs $\left\{m_{p, s}, s=1, \ldots, S\right\}$ and the set of BBAs $\left\{m_{c, s}, s=1, \ldots, S\right\}$ before making their PCR5 combination.

\section{CONCLUSIONS}

In this work we have proved that any dichotomous basic belief assignment (nondogmatic, or dogmatic) can be decomposed into two simpler proper belief assignments called the pro-evidence and contra-evidence that can be combined with PCR5 rule to retrieve the original BBA. This canonical decomposition is unique and is always possible. No simple explicit form of the expression of the solution exists but the solution can be found quite easily with numerical solvers (Matlab ${ }^{\mathrm{TM}}$, Maple ${ }^{\mathrm{TM}}$, etc). We have also shown that the decomposition of any dichotomous basic belief assignment cannot be done in all the cases with other well-known rules of combination, which reinforce the interest of PCR5 principle for belief function combination. This PCR5-based canonical decomposition allows also to establish the notion of internal conflict of a dichotomous source of evidence which could be helpful in some applications. It offers the possibility to combine several dichotomous sources of evidence based on the fusion of their canonical components. This will be presented in details in a forthcoming publication. The open challenging question is how to extend this notion of canonical decomposition for working with more general basic belief assignments to make their combination more effective (if possible), and how could we define a measure of (uncertain) information thanks to this canonical decomposition.

\section{ACKNOWLEDGEMENT}

Jean Dezert is very grateful to Professor Christine Marois of the Faculté de Droit d'Économie et de Gestion of Orléans University, France for her inspiration and valuable help for the establishment of the concise proof of the theorem 1.

\section{References}

1. Shafer G. A Mathematical Theory of Evidence, Princeton Univ. Press, 1976.

2. Smarandache F, Dezert J. (Editors). Advances and applications of DSmT for information fusion, American Research Press, 2004-2015; Vols. 1-4. http://www.onera.fr/staff/jean-dezert?page=2

3. Smets P. The canonical decomposition of a weighted belief, Int. Joint Conf. on Artificial Intelligence, Morgan Kaufman, San Mateo, CA, USA, 1995; 18961901.

4. Dezert J., Wang P., Tchamova A. On the validity of Dempster-Shafer theory, Proc. of Fusion 2012, Singapore, July 9-12, 2012. 
5. Tchamova A., Dezert J. On the behavior of Dempster's Rule of combination and the foundations of Dempster-Shafer theory, Proc. of 6th IEEE Int. Conf. on Int. Syst., Sofia, Bulgaria, Sept. 6-8, 2012.

6. Dezert J., Tchamova A. On the validity of Dempster's fusion rule and its interpretation as a generalization of Bayesian fusion rule, Int. J. of Intelligent Syst., March 2014; 29(3): 223-252.

7. F. Smarandache, J. Dezert, On the consistency of PCR6 with the averaging rule and its application to probability estimation, Proc. of Fusion 2013, Istanbul, Turkey, July 2013.

8. F. Smarandache, J. Dezert, Information Fusion Based on New Proportional Conflict Redistribution Rules, in Proc. of the 8th Int. Conf. on Information Fusion, Philadelphia, USA, 25-29 July, 2005.

9. Dezert J., Smarandache F. Non Bayesian conditioning and deconditioning, International Workshop on Belief Functions, Brest, France, April 2010.

10. Smarandache F., Dezert J., Tacnet J.-M. Fusion of sources of evidence with different importances and reliabilities, in Proc. of Fusion 2010 conference, Edinburgh, UK, July 2010.

11. https://bfasociety.org/

12. Denœux T. Conjunctive and disjunctive combination of belief functions induced by nondistinct bodies of evidence, Artificial Intelligence, 2008; 172: 234-264.

13. Yager R., On the Dempster-Shafer framework and new combination rules, Information Sciences, 1987; 41: 93-138.

14. Dubois D., Prade H. Representation and combination of uncertainty with belief functions and possibility measures, Computational Intelligence, 1988; 4: 244264.

15. Weisstein E. Quartic Equation. From MathWorld-A Wolfram Web Resource. http://mathworld.wolfram.com/QuarticEquation.html

16. Zadeh L.A. On the validity of Dempster's rule of combination, ERL Memo M79/24, Department of EECS, Univ. of California, Berkeley, U.S.A., 1979.

17. Zadeh L.A. A simple view of the Dempster-Shafer theory of evidence and its implication for the rule of combination, The Al Magazine, 1986; 7(2): 85-90. 